\title{
Research and implementation of educational management system based on role access control technology
}

\author{
Li Qingsheng $^{1}$ and Zhao Lijun ${ }^{2}$ \\ ${ }^{1}$ Academic Affairs, Chengde Petroleum College, Chengde, China \\ ${ }^{2}$ Electrical and Electronic Engineering Department, Chengde Petroleum College, Chengde, China \\ cdpc13065@163.com
}

\begin{abstract}
Keywords: Role access control, Adaptive access control model, Educational management system, Data access control

Abstract: In this paper based on the role access control model has the characteristics of network security, and the adaptive data access control model is proposed to realize the authorization mechanism of educational management system in universities. Adaptive data access control model is mainly used for the effective allocation of user permissions in educational management system, and the allocation of permissions is mainly related to the user, role, and allows the implementation of access operations and other key links. The results show that the access control mechanism provides higher flexibility for the system under the premise of ensuring the security of the system operation.
\end{abstract}

\section{Introduction}

In recent years, based on the computer information processing technology, colleges and universities in our country have established the digital characteristic of the university educational management. With the continuous development of information technology, the continuous expansion of the colleges scale and continued rise in the number of college students, the work of educational management system is becoming more and more complicated, the existing educational management system has been unable to satisfied the increasing all kinds of demand[1]. Therefore, in order to meet the needs of the reform of educational management system in our country, it is urgent to improve the system function with more advanced technology, and it is a solid foundation for the safety, reliability, high efficiency and flexibility of the management system.

System has a lot of users and resources, it leading to the university educational management system security control of the necessity and complexity. Traditional access control type-Discretionary Access Control (DAC) and Mandatory Access Control (MAC) have been unable to meet the needs of modern information system security. In this case, the Role-Based Access Control (RBAC) technology has emerged[2]. RBAC is a method to allow or restrict access to the ability and scope through the introduction of the role concept, and in the RBAC model based on the proposed system adaptive data access control model to achieve user authorization mechanism, so that the user's management and maintenance work can be simplified.

\section{The university educational management system}

Because of the large number of users in the university educational management system, the authority management is complex. Therefore, in order to adapt to the different members of the college and university, the educational management system uses the adaptive access control strategy to realize. Under the premise of effective guarantee system security, the system satisfied the user's access control requirements. 
The main business logic of university educational management system is shown in Fig. 1.

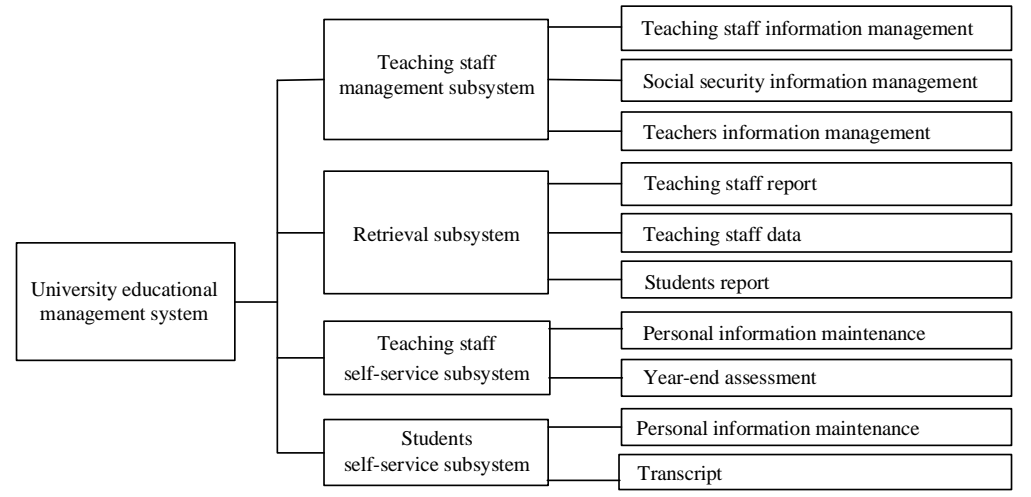

Fig.1 The main business logic of university educational management system

University educational management data relationship is significant, so the user's access control authority for effective management is to ensure the system safety of the premise. In order to satisfy the user requirements, the system uses access control adaptive management mechanism in the implementation process. It can avoid manual intervention and guarantee the security of the system, and realize the adaptive management of user and user authorization.

\section{Access control model}

The Role-Based Access Control. The Role-Based Access Control (RBAC) has become the mainstream of the current access control technology, is considered to be the most potential access control strategy. The basic idea of RBAC model is the concept of role between the user and the authority, the system first permissions assigned to the role, the user access to the role to obtain the corresponding access rights. Role is the core element of the role based access control, the user's authority is described by the role, and the role is a collection of permissions. Typical RBAC models such as the RBAC97 model is shown in Fig. 2.

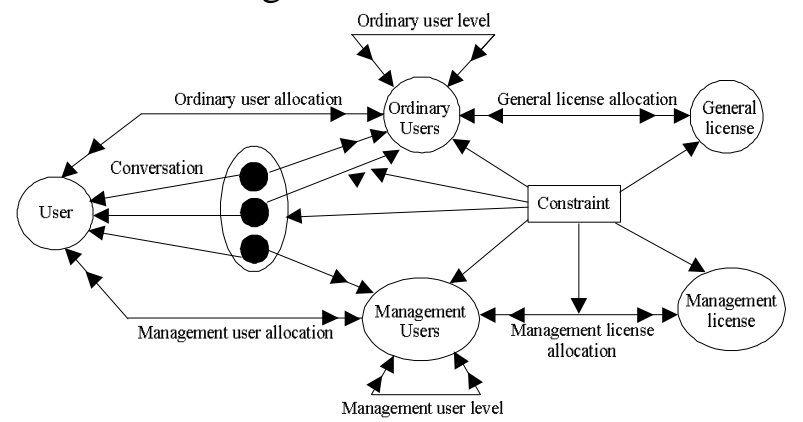

Fig. 2 The RBAC97 model

The model gives full play to the hierarchical management mechanism of RBAC, in which users can have different levels of management authority. At the same time, with the different management level, the corresponding management work also reflects the abstract to the concrete process.

Adaptive access control model. The access control adaptive model is based on RBAC, which includes the adaptive authorization management and user management module, and RBAC is the main access control. The authorization principle in the access control mechanism is closely related to the user and business logic[3-5]. The system is designed by the adaptive model shown in Fig. 3.

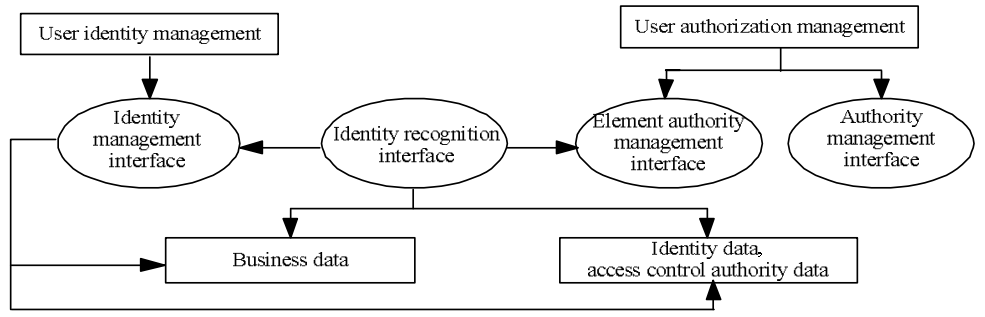

Fig. 3 The adaptive model 
In Fig. 3, in order to enable the system to achieve adaptive user identity management and identification, increases the user identification and management interfaces. Different from the traditional access control model, the user management system of educational management system, user management, log management, real-time authorization management, user registration and distribution system are based on business logic data adaptive management mode, so as to realize the real-time performance of the authorization. Adaptive access control management process is shown in Fig. 4.

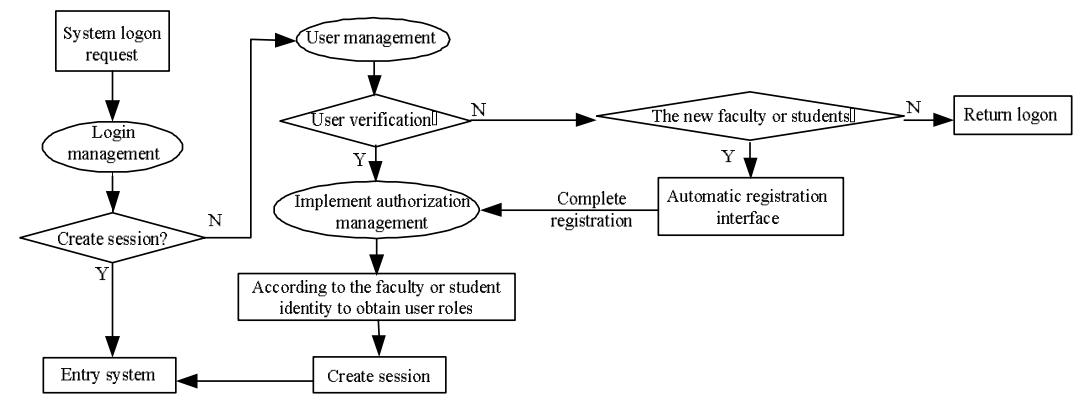

Fig. 4 Adaptive access control management process

After the user successfully login in to the system, the access behavior constraint module will play an important role in the whole system behavior and user behavior. Thus, effectively avoid the illegal operation of the user. First, the system's access behavior control management mechanism checks the legitimacy of the user's business logic request. Secondly, if the business data information is ready, the system interface module before the data provided to the user, then to create a visual interface based on the access level, and ultimately to provide users with the corresponding business operations.

\section{Realization of adaptive access control educational management system}

System login management. The university educational management system of the user login mechanism is used the conventional account and password mode. It is different from other systems, the user login system also can realize the user's management, maintenance and the corresponding authorization management. The user login process is shown in Fig. 5.

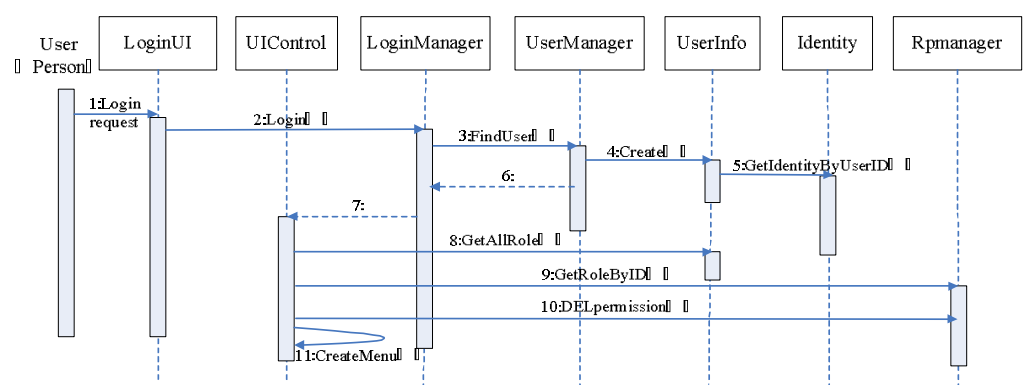

Fig.5 The user login process

License and role management. This system is based on RPManager to complete the user's license and role information maintenance and update (as shown in Fig. 6). The model does not need to create data for each user, it can save the system overhead, and can be in more than one user to modify the license information and role information, to ensure the uniqueness of the data.
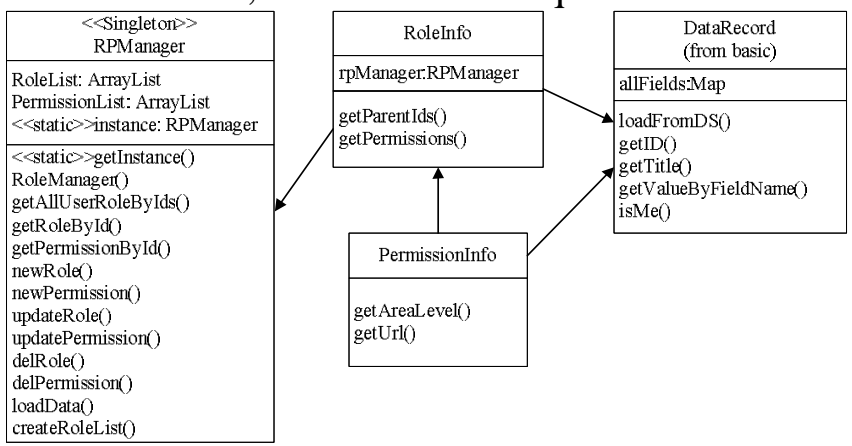

Fig. 6 System license and role management 
PermissionInfo and RoleInfo are the auxiliary class RPManager, which function is to record a single license or role information. In the process of retrieving, RoleInfo also has the attribute of pointing to the instance of RPManager. From the result of RPManager, the real license and the role data are obtained.

Visualization access control. The management mechanism can create the mapping relationship between the position, the department and the user role. The user identity management interface of the system is shown in Fig. 7. The implementation of the system user access control management interface is shown in Fig. 8.

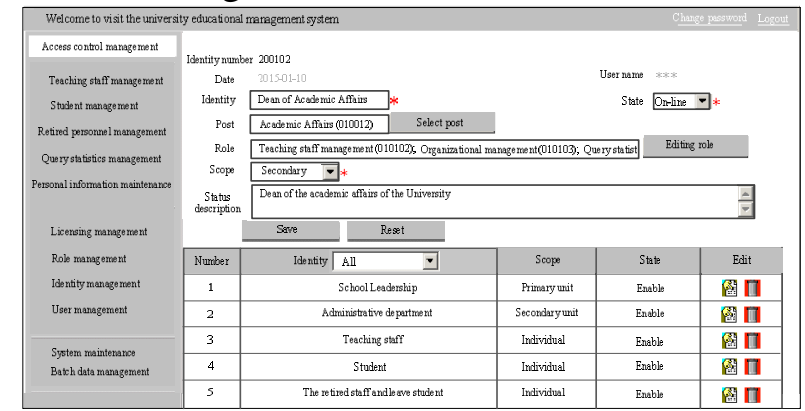

Fig. 7 The user identity management interface

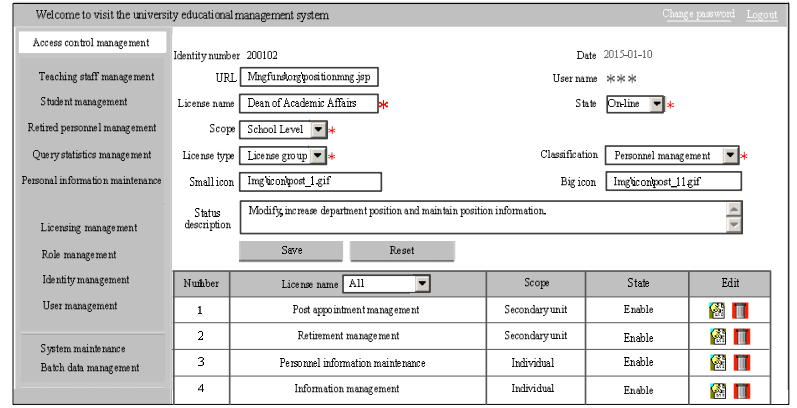

Fig. 8 The system user access control management interface

It can be seen from Fig. 8, the use of access control mechanisms to ensure that the system of different personnel to different roles and operating rights, through this way to effectively ensure the system security.

\section{Summary}

In this paper, in order to solve the safety design requirements of the educational management system, the role model based on the characteristics of the access control, the access control model is constructed to improve the data security, reliability and flexibility. At the same time, the design scheme of adaptive access control for different users in the process of implementation is given. The results show that the access control mechanism can provide more flexibility for the system, so that the management and maintenance of the user can be simplified.

\section{References}

[1] Li Qingsheng. Research and implementation of the relevant technology of educational management system in Universities. Hebei: Yanshan University. 2012.

[2] Liu Xiaochen. The trusted platform of network access control technology research and application. Jiangsu: Nanjing University of Science and Technology.2011

[3] Xiong Houren, Chen Xingyuan, Zhang Bin. Security Principles for RBAC-based Authorization Management. Computer Science. 2015, 42(3): 117-123

[4] Lin Qingguo, Liu Yanbing. A trust-based dynamic access control scheme. Journal of Chongqing University of posts and telecommunications(Natural Science Edition) . 2010, 22(4): 478-482

[5] Sun Ling, Xin Yan, Luo Changyuan. Pervasive computing access control model based on extended RBAC. Journal of Computer Applications. 2010, 30(4): 1045-1052. 